Short Communication

\section{Modeling of A.I based Inhalation for Advanced Life Support System Development}

\author{
Md. Sadique Shaikh ${ }^{1 *}$ and Tanvir Begum² \\ 'ISTM Mumbai, M.S, India \\ ${ }^{2} \mathrm{H}$, Maveen Pvt. Ltd. Jalgaon, M.S, India
}

\section{Abstract}

Present piece of idea exhibits to divert attention towards automated high precision Life Support System (LSS) instead of manual one using medical intelligence devices while treating and diagnosis to the patient, where Ventilator, inhaler and respiratory control is most important factor during operation, surgeries and in other likewise medical emergency situations to maintain proper saturation in patient lungs to sustain their lives. This work gives idea, how we can design A.I based Inhaler System for the same.

\section{Modeling}

The below model depicted the successful engineering how one can design and implement A.I based Inhaler System for Respiratory precision to maintain saturation of human breathing system, we can even labeled the system as Artificial Intelligence based lungs or Lungs Support System or Oxygen Support System or Breath Support System but purpose remains same (Figure 1).

Patient respiratory sensed and input parameters send to Saturation Measurement Unit which must be highly calibrated and compare Measured saturation with Standard Saturation to find deviation for error detection and correction from where physical quantity fed to A.I based processing \& controlling chamber with time control for inhalation decision support with all expert analysis and diagnosis which generate electronic respiratory report with necessary timing,

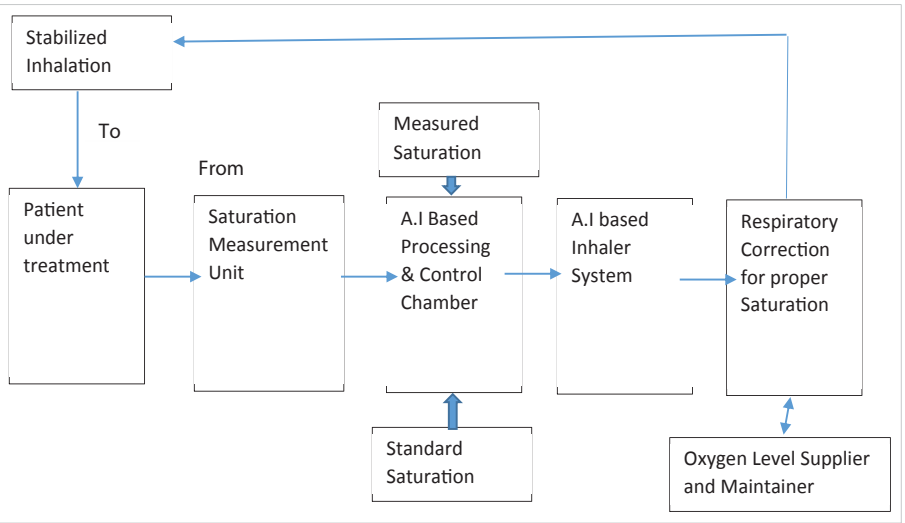

Figure 1: Source: Prof. (Dr.) Md. Sadique Shaikh \& Tanveer Begum.

\section{More Information}

*Address for Correspondence: Dr. Md. Sadique Shaikh, Professor, 12, Kazi Plot, Bhusawal 425201, M.S, India, Tel: 9923361978 . Email: sids_nsk@rediffmail.com

Submitted: 16 October 2019

Approved: 26 November 2019

Published: 27 November 2019

How to cite this article: Shaikh MS, Begum T. Modeling of A.I based Inhalation for Advanced Life Support System Development. Int J Phys Res Appl. 2019; 2: 049-050.

DOI: dx.doi.org/10.29328/journal.ijpra.1001015 Copyright: () 2019 Shaikh MS, et al. This is an open access article distributed under the Creative Commons Attribution License, which permits unrestricted use, distribution, and reproduction in any medium, provided the original work is properly cited.

Keywords: A.I based Inhaler system; A.I based life-support system; Medical robotics; Surgical robots

\section{Check for updates}

processing and control signals to get follow further send to A.I based Inhaler System to decide and fixed precision parameters for respiratory. This report A.I based processing and control chamber generate on the basis of actual saturation and required saturation facts and figures further passed to Respiratory Correction for Proper Saturation Unit which is cascaded with Oxygen Level Supplier and Maintainer and work integrated. Finally stable inhalation feedback to patient for life survival in critical condition [1-6].

\section{Limitation}

The limitation of the purposed model is its need Bionic or DeepMind processor with high precision processing ability to develop electronic report of medical intelligence. Hence purposed piece of research also depend majorly on the success of Bionic and DeepMind processors and after the success of them further mentioned technology form. To develop and implement such an advance Life Support System would be great victory of medical science.

\section{Conclusion}

This piece of research gives your idea, how to engineer and implement precise artificial Intelligence based Inhaler System which is one of the most promising, needful and social welfare requirement to save patients' lives with excellent Life Support System (LSS). 


\section{Acknowledgement}

I would deeply acknowledge this piece of work to Safeena Shaikh, my loving sons Md. Nameer Shaikh and Md. Shadaan Shaikh. Equally I want to acknowledge this work to my forever loving friend and co-author of this work Tanveer Sayyed and to sweet family.

\section{References}

1. NSF/EC Understanding on Co-operation in Information Technologies. Strategic Research Workshops IST-1999-12077

2. Shaikh MS. Analysis and modeling of Strong A.I to engineer BIONIC brain for humanoid robotics application. Am J Embedded Sys Appli. Published by Science Publishing Group. 2013; 1: 27-36.

3. Shaikh MS. Ultra Artificial Intelligence (UAl): Redefing Al fir New Research Dimension. Advanced Robotics \& Automation (ARA), OMICS International, London. 2017; 6: 1-3.

4. Shaikh MS. Fundamental Engineering for Brain-Computer Interfacing $(\mathrm{BCl})$ : Initiative for Neuron-Command Operating Devices. Computational Biology and Bioinformatics (CBB). Science PG. 2017; 5: 50-56.

5. Shaikh MS. Defining ultra-artificial intelligence (UAI) implementation using bionic (biological-like-electronics) brain engineering insight. MOJ App Bio Biomech. 2018; 2: 127-128.

6. Shaikh MS. Insight Artificial to Cyborg Intelligence Modeling. Arch Ind Engg. 1: 1- 5. 